\title{
Modification of nanocrystalline cellulose for bioactive loaded films
}

\author{
Paula Criado ${ }^{1}$, Carole Fraschini ${ }^{2}$, Stéphane Salmieri ${ }^{1}$ and Monique Lacroix ${ }^{1 *}$ \\ ${ }^{1}$ Research
}

Laboratories in Sciences Applied to Food, Canadian Irradiation Centre (CIC), INRS-Institute Armand-Frappier, University of Quebec, 531 Boulevard des Prairies, Laval, Quebec, H7V 1B7, Canada

${ }^{2}$ FPInnovations, 570 boulevard Saint Jean, Pointe-Claire, Quebec, H9R 3J9, Canada.

\begin{abstract}
Despite the use of petrochemical derived packaging, many problems such as browning and food spoilage still happen in food after harvesting. There is an increasing consumers concern for food shelf life to be extended as much as possible along with a big interest in green and bioactive materials, that could be used in direct contact with aliments. In order to reach public demand, biopolymers coming from natural sources such as plants or animals have been used to replace synthetic materials. Even though natural polymers are biodegradable, they don't reach regulations required with respect to mechanical properties in commercial applications. However, the mechanical properties can be improved when reinforced with nanoparticles. Several reinforcing polymers such as clays, silica or silver have been used for industrial applications, but cellulose nanocrystals (CNCs) are a better choice for food industry due to their biodegradable and biocompatible nature as well as their outstanding potential in improving mechanical and barrier properties of nanocomposites. CNCs consist of anhydroglucopyranose units (AGU) linked together and several functional hydroxyl groups found on its surface. Modifications of the CNC surface chemistry can give to cellulose new functionalities that open the way to the development of new bioactive reinforcement in food packaging. The present review will be focused on covalent and non covalent modifications that can be achieved on surface $\mathrm{CNC}$ with the aim of adding functionalities to be applied for food industry.
\end{abstract}

Keywords: food packaging, cellulose nanocrystals, CNC, acetylation, polymer grafting, TEMPO oxidation, layer-by-layer, cationic surfactants, radiation-induced polymer grafting.

\footnotetext{
* Author to whom correspondence should be addressed, Telephone: +1-450-687-5010; Fax: +1-450-686-5501; E-mail: monique.lacroix@iaf.inrs.ca
} 


\section{Introduction}

37 Nowadays, consumer demand focuses on product shelf life [1], suppression of apparition of undesirable and uncomfortable flavors and odors produced after a few days of storage [2]. Consumer interests in the addition of ingredients which can provide beneficial effects for food quality and health is also increasing [3]. Due to the increase of consumer demand, bioactive packaging has been proposed with the aim of remaining cost-effective and healthy for consumption [4-6].

Functional biodegradable films have been implemented, for instance, an antimicrobial packaging can be used as a retardant for microbiological proliferation [7] on fresh food. Other functional packaging can be developed to avoid problems such as food browning, discolorations and microbial spoilage.

Currently, scientists challenge lies on the use of active biopolymers such as chitosan, which has the potential to preserve and protect from antimicrobial attack in food coatings [8]. However, the use of chitosan as a biodegradable film has some limitations such as poor vapor barrier, weak mechanical properties [4] and also antimicrobial limitations when it is used as insoluble films [9]. In order to improve the functionality of chitosan based films, addition of active reinforcements has been proposed. Several composites have been developed by adding reinforcement agents to polymers in order to enhance their thermal, mechanical and barrier properties [10]. A uniform dispersion of these reinforcement particles in polymer matrices can lead to a better molecular mobility, relaxation behavior and the consequent thermal and mechanical properties of the material [10]. According to Suyatma et al.[11] a reinforcing agent increases the physico-chemical properties, acting as a lubricant in a polymer network. Taking into account that polymer-polymer interactions within polymer chains are made of hydrogen bonding and van der Waals forces, a reinforcing agent role is to break down these bonds and increase the flexibility of the polymer network. Ludueña et al. [12] have demonstrated that the smaller the filler particles loaded in polymer matrices, the better the interaction in the polymer network and the higher is the cost-price efficiency. In this context several nanoreinforcements have been interesting due to their high surface that provides better reinforcement effects [13-17].

In addition to the enhancement of mechanical and barrier properties given by nanoreinforcements, there other several functionalities, for instance antimicrobial and antioxidant activity that can be to the properties of the nanoreinforcements when they are used for packaging systems. Some added properties can be achieved by covalent or noncovalent modification of reinforcements based on polymer modifications presented in literature. The aim of these new type of nanoreinforcements is to have "smart" properties with applications of food packaging fields.

\section{Nanocomposites}

Nanoparticles have a great utility in biopolymer formulations for food preservation. In this context, nanoparticles or nanoreinforcements are polymeric fillers which are characterized by having one dimension in the nanometric range [18]. Thus, these nanosized inorganic or organic fillers come with various geometries (fibers, flakes, spheres, particulates) [4] 
Fillers can be classified in three categories, depending on whether the dimensions are in the nanometric range or not. Spherical structures such as silica, nanotubes or nanocrystals can be found in a wide dimensional range [10], but only those found nanometrical, will be considered as a nanocomposite [18]. Several types of nanocomposites are found in industrial applications, for instance Polymer-Clay-Nanocomposites (PCN) are used in food packaging [17]. However, there is a coming interest on cellulosic nanoparticles due to its abundant organic source and biodegradability and light weightiness. Major interest has been found in using cellulosic materials as the main components in the manufacture of biodegradable packaging materials [19-21], in addition to the stimulating search for nonpetroleum-based structural materials [22]. Several sizes of cellulose can be found like fibers, cellulose microcrystals and cellulose nanocrystals which are obtained by physical and chemical modifications of cellulose. Indeed, cellulose nanocrystals are extracted by via chemical treatment with strong acids and the result is the cellulose within shape of nanoparticles which surface is characterized of functional hydroxyl groups which allow $\mathrm{CNC}$ to be soluble in water. Modifications of $\mathrm{CNC}$ such as grafting active polymers, change of solubility and/or anionic charge can be carried on to give some functional properties that can be applied for several purposes.

In this review, technological applications of biodegradable food packaging based on modified cellulose will be discussed. After a brief introduction to cellulose characteristics and its derivatives, cellulose nanocrystals (CNC) will be defined for nanocomposites purposes in bioactive films.

\section{Cellulose-based nanoreinforcements}

Cellulose is an organic polymer known to occur in a wide variety of living species from the world of plants, bacteria and animals. Cellulose structure consists of a linear homopolymer of $\beta$-D-glucopyranose units which are linked together by $(1 \rightarrow 4)$-glycosidic bonds [17]. The degree of polymerization (DP) can be up to 10,000 - 15,000 [23]. Compared to inorganic nanoreinforcements, cellulose has a positive impact in industrial applications because of its advantages listed below [24]:

- renewable nature

- low cost

- low energy consumption

- easy disposal by combustion

- environmental acceptance

- wide variety available worldwide

- high specific strength and modulus

- comparatively easy processability due to their nonabrasive nature

- relatively reactive surface, which can be used for grafting specific groups.

Cellulose chains are aggregated microfibrils which contains amorphous and crystalline regions. Habibi et al. [25] described the amorphous parts as chain dislocations along the fiber contrary to the crystalline region where cellulose chains are tightly packed and linked by a strong and very complex intra-and intermolecular hydrogenbond network. Crystalline isolation from cellulose fibers was developed in 1950, when Ränby et al. [26] reported the 
first sulfuric acid-catalyzed degradation of cellulose fibers. Since then, acid hydrolysis has been applied to obtain nanocristalline cellulose [25]. The obtained crystals keep a similar crystallinity as that presented in the original cellulose fibers. However, dimensions can vary depending on the source of the cellulose. For instance, CNC from wood are 3-5 nm in width and 100-200 nm in length, while those obtained from Valonia, a sea plant, can be up to $20 \mathrm{~nm}$ in width and 1000-2000 $\mathrm{nm}$ in length [25].

Cellulose nanocrystals are mostly extracted from plant cells walls, but it can also be found in bacteria, algae or animals. However, it is shown that lignocellulosic derived nanocrystal provide a higher mechanical strength and high mechanical strength-to-weight ratio compared to other type of cellulose [22].

Because of its larger surface area to volume ratio, large amounts of bioactive molecules are more likely to be attached to the cellulose surface due to high number of hydroxyl groups available on its surface. This is the reason why, cellulose nanocrystals have been actively investigated as a potential nanocomposite in material development $[4,14,16,24,27,28]$.

\section{Cellulose Nanocrystals (CNCs)}

The main process of isolation of CNCs is based on acid hydrolysis of cellulose with concentrated sulfuric acid at different temperatures [25]. The resulting nanocrystals exhibit negative sulfate ester groups attached onto the CNC surface, thus, providing a colloidal stability in aqueous medium. Another process that has been found in literature [29-31]is TEMPO-mediated oxidation, which consists in a surface modification of the primary hydroxymethyl groups into a negatively charged carboxylic groups. The modified CNC also form a homogenous suspension when dispersed in water due to the presence of negatives charges.

Nowadays, researchers actively work on finding better ways to provide food quality and beneficial health effects in this field. In this context hydrophobic components such as essential oils, organic acid solvents additives or plant extracts have been added to food coatings in order to avoid microbiological growth, thus, extending product shelf life [3236]. It is found that others structurally similar polymers have been chemically modified to give functional properties. This is the case of antioxidant activities added to chitosan and gelatin explained by the research group Curcio et al. [37] and Spizzirri et al. [38,39]. Reactive oxygen species (ROS) and oxygen-derived free radicals are the resulting components of a biological oxidation that may contribute to pathological effects, such as diseases and cellular degeneration, including aging, cancer and diabetes [40,41]. Even though, the human body produces antioxidant that can retard this process, it is not sufficient to prevent it from the entire damage $[42,43]$. If modification is performed onto $\mathrm{CNC}$ surface, interesting antioxidant properties can be added to food coating in order to prevent food rancidity and fast aging.

It may be noted that $\mathrm{CNC}$ has the advantage of having an abundance of hydroxyl groups at its surface, thus, chemical modifications of these functional sites could be performed. The modifications suggested in this review will allow CNC to enhance its compatibility and dispersibility with other compounds such as antioxidants and antimicrobials essentials oils, 
as well as, hydrophobic polymers. Results of modifications have the purpose of spreading nanocellulose applications for food industry or other reliable fields.

\section{Surface modifications of CNC}

\section{Acetylation}

Due to the hydrophilic behavior of cellulose nanocrystals, there is an interest to improve its compatibility with hydrophobic media. Acetylation is a reaction that allows the interaction of hydroxyl groups $(\mathrm{OH})$ with acetyl moieties. Studies reported $[22,44]$ that a reaction of esterification can make cellulose more hydrophobic. Indeed, available hydroxyl groups on the cellulose surface can react with acid anhydride or acyl chloride reagents. Figure 1 shows a schema illustrating a suggested mechanism of acetylation of CNC. Jonoobi et al. [44] indicated that the rate of acetylation is low when cellulose has strong hydrogen bonding interactions. Results showed a higher degree of substitution (DS) of hydroxyl groups in kenaf fibers compared with that of nanofibers .

Another mechanism of acetylation can be carried out using a heterogeneous process. In this process, cellulose is first swollen in a diluent such as toluene, benzene or amyl acetate and is then acetylated with acetic anhydride in the presence of the catalyst sulfuric acid [45]. It has been found that cellulose morphology can vary with the chosen acetylation method. It has been observed that heterogeneous acetylated crystals remain morphologically intact [46]. Ultrastructural aspects on acetylation of cellulose reported by Sassi and Chanzy [45] shows that heterogeneous acetate modified cellulose surface chains surround the nonmodified cellulose core. In opposition, homogeneous process leads to substantial morphological changes caused by a constant stripping of the cellulose surface chains as they become acetylated and soluble in the acetylating medium.

With the objective of reducing the number of laborious steps in modification of nanocellulose, Braun and Dorgan [47] proposed a one-step procedure which consists in a fisher esterification of hydroxyl groups of cellulose simultaneously with hydrolysis of the amorphous cellulose. Surface functionalized cellulose nanocrystals are the result of the reaction using a mixture of an organic acid (acetic or butyric acid) and hydrochloric acid. FTIR spectroscopy showed the presence of ester functional groups and multiangle laserlight scattering (MALLS) indicated that half of the hydroxyl moieties were substituted [47]. Moreover, a high dispersibility is achieved when immersing the final product in ethyl acetate or toluene solution.

Thereby, these methods lead to a promising dispersion of nanocellulose into hydrophobic polymers by acetylation of hydroxyl moieties of CNC surface or by one-step modification of amorphous cellulose.

\section{Polymer grafting}

Grafting polymerization is a well-known method to develop material with particular structure and properties [38]. Polymer grafting is divided into two approaches, the "grafting-onto" and "grafting-from" [48]. The "grafting-onto" approach considers the 
attachment of pre-synthesized polymer onto the available hydroxyl groups on the cellulose by using a coupling agent. On the other hand, the "grafting-from" approach involves the polymerization in situ from initiators attached to the macromolecule surface. New functionalized cellulose nanocrystals can be inserted into a polymer matrix in order to develop new smart and biodegradable materials.

Biological applications for food industry were suggested by Spizzirri et al. (2009) and Curcio et al. ( 2009) by attaching antioxidants onto polysaccharides surface by free radical grafting methods. Based on the beneficial effects of antioxidant on human health and food preservation [49], catechin (CT) and gallic acid (GA) were used in order to synthesize an antioxidant-gelatin conjugate. In this context, antioxidant activities of GA and CT grafted onto gelatin were compared by a process using a hydrogen peroxide-ascorbic acid redox pair as the initiator. Synthesis of antioxidant gelatin was performed by preparing a solution $1 \%,(\mathrm{w} / \mathrm{v})$ of gelatin dissolved in water, then, $5.0 \mathrm{mmol}$ of hydrogen peroxide and $1.4 \mathrm{mmol}$ of ascorbic acid were added. The mixture was maintained at $25^{\circ} \mathrm{C}$ and after $2 \mathrm{~h}$ the antioxidant was added to the solution. The reaction of gelatin solution and antioxidant was maintained for $24 \mathrm{~h}$ [38]. Characterization of antioxidant-gelatin conjugate was performed by UV-vis spectroscopy in order to observe the changes in the structure of the polymer. Results showed two characteristic peaks at 227 and $272 \mathrm{~nm}$ related to the presence of GA and CT. Similar results were obtained by Curcio et al. [37] when grafting GA and CT onto chitosan. Determination of antiradical properties were measured by scavenging effect of 2,2'-diphenyl-1-picrylhydrazyl (DPPH) radical [50] and obtaining high scavenging activities of $66 \pm 3 \%$ and $98 \pm 3 \%$ for GA and CT, respectively were observed.

All the previous methods can be applied to CNC, a possible mechanism of conjugation of an antioxidant molecule by the free radical grafting has been proposed in Fig. 2 . Modifications of CNC could lead to enlrge the functionalities of this good mechanical reinforcement to a biactif/antioxidant properties.

Nanocomposite films of maleated polypropylene grafted cellulose (PPgMA) nanocrystals and surfactant modified nanocrystals dispersed in an amorphous matrix of atactic polypropylene were analyzed by Ljungberg et al. [51]. The resulting modified film showed tensile strength improvement compared to that of the neat polypropylene matrix. Similar results were observed by Cao et al. [52] who reported that in situ polymerization of presynthesized waterborne polyurethane (WPU) on the surface of cellulose nanocrystals induces a co-crystallization. Hence, this phenomenon leads to a co-continuous phase between the matrix and filler which enhances the thermal stability and mechanical strength of the resulting nanocomposites in food packaging.

\section{RAFT surface polymerization}

Reversible Addition-Fragmentation chain Transfer (RAFT) is a recent technique for free radical polymerization. This method has the advantage to control the molecular weight and the topology of the polymer grafted onto another polymer backbone [53]. With the aim of ensuring an efficient molecular distribution that leads to an increased antimicrobial activity, it has been chosen to graft antimicrobial long chain polymers onto cellulose fiber via RAFT polymerization. In this context, quaternary ammonium compounds (QACs) are cationic polymers with several advantages. These include their antibacterial activity, low toxicity, 
low tissue irritation, increased efficiency and selectivity, and prolonged lifetime [54,55]. In addition, working with long chain antimicrobial agents gives to the binding polymer a higher positive charge which is expected to better attach to negatively charged bacteria rather than using monomeric or low molecular weight cationic components [53]. The general mechanism of attachment of these quaternary ammonium compounds on bacteria is characterized by 4 possible effects: (i) adsorption of positively charged QACs on the negatively charged cell surfaces of microorganisms, (ii) compatibility of lipid bilayer bacterial cytoplasmic membrane with the hydrophilic chains of the QAC-polymer, (iii) binding to the cytoplasmic membrane and (iv) disruption of the cytoplasmic membrane. The instability and the loss of cytoplasmic constituents will lead to the death of microorganisms $[55,56]$.

Hence, Roy et al. [53] have suggested to use tertiary amino groups of 2-(dimethylamino) ethyl methacrylate (DMAEMA) polymer grafted onto cellulose surface. Cellulose-gPDMAEMA was then quaternized by alkyl bromide action, exhibiting a high activity against Escherichia coli. The group observed an influence of the grafting ratio of alkyl chain length (C8-C16), the hydrophobicity of the sample and the degree of quaternization. High efficacy against $E$. coli was found in tertiary cellulose-g-PDMAEMA as well as in quaternized cellulose. The group reported that a grafted ratio of $27 \%$ of non-quaternized PDMAEMA and quaternized with C8 alkyl chains led to a 350 and $<100 \mathrm{CFU} / \mathrm{mL}$ compared to $1 \times 10^{6} \mathrm{CFU} / \mathrm{mL}$ bacteria added to the sample. The decrease of antibacterial activity with the increase in alkyl chain length has been discussed by other authors [57-60]. Indeed, an optimal alkyl chain of eight carbon atoms may lead to a strong interaction of the antimicrobial agent with the bacteria. Moreover, increasing the hydrophilicity (9-27\% grafted PDMAEDA) favored the interaction of the polymer with the bacteria, thus, reaching their cytoplasmic membrane [58].

Antimicrobial polymers were also obtained by quaternization of the poly(2dimethylamino)ethyl methacrylate-co-oligo(ethylene glycol) methyl ether methacrylate P(DMAEMA-co-OEGMA) [61] against Gram-positive bacteria Bacillus subtilis. Similar correlation of the importance of hydrophobicity was found by these authors in the minimum inhibitory concentration (MIC). This innovative technique has also been applied at room temperature using $\gamma$-irradiation as the source initiator. Well-defined polymers onto existing surface have been achieved with a narrow polydispersity $[62,63]$.

\section{TEMPO-mediated oxidation}

Nowadays, a new selective and more efficient oxidation method has been developed for cellulose fibers. Reagent known as 2,2,6,6-tretramethylpiperidine-1-oxyl (TEMPO) has been used to oxidize the surface hydroxyl groups of $\mathrm{CNC}$ into carboxylic groups $[25,30,31,64]$. The TEMPO-mediated oxidation is an alternate promising route to convert surface hydroxyl of cellulose into charged carboxyl entities. The mechanism of TEMPOoxidation is considered as a green and simple technique [25] to modify the surface of macromolecules.

TEMPO-based chemical modification has a selectivity to oxidize cellulose surface, leaving intact the hydroxyl groups of cellulose core. Since the first work of de Nooy et al. [29] it 
was demonstrated that the oxidation was highly selective with $98 \%$ of the primary hydroxyl of potato starch in cold water and $>90 \%$ in the case of Dahlia inulin at $\mathrm{pH} 10.5-11$. TEMPO-mediated oxidation was also applied to tunicin whiskers with the objective of converting surface hydroxyls of cellulose into negatively charged carboxyl entities [30]. Moreover, the authors reported that this technique leads to more stable suspensions of cellulose whiskers compared to aqueous suspensions of CNC extracted with sulfuric acid. Concerning the morphology of the chemically modified cellulose, Habibi et al. [30] concluded, after examination in transmission electron microscopy (TEM), that TEMPOoxidated tunicin whiskers kept the same distribution and cristallinity than those of native cellulose whiskers.

Stability and non-flocculation was explained by the presence of negative charges at the surface of cellulose, thus a better individualization of the crystallites [64]. With respect to the crystal size of cotton linters and microfibrils of parenchyma cell cellulose (PCC), Montanari et al. [64] have shown a decrease of crystal size and a degradation of the amorphous areas of the starting material. Comparing different $\mathrm{HCl}$ and TEMPO hydrolysis, it was found that the degree of oxidation (DO) achieved for PCC microfibrils and cotton linters after TEMPO-oxidation was 0.4 and 0.23 , respectively. On the contrary, $\mathrm{HCl}-$ hydrolysis oxidation reached the DO values of 0.23 for PCC microfibrils and 0.15 for cotton linters.

It is important to highlight that TEMPO-oxidation can be also used as an intermediate reaction for polymer grafting taking advantage of the negative charge and radical formation in carboxylate groups. Poly(ethylene glycol) (PEG) grafting onto cellulose was conducted via TEMPO-oxidation by Araki et al. [65]. A quantity of 0.2-0.3g of resulted PEG was grafted per gram of cellulose and a sterically stabilized aqueous suspension was obtained. The fact of having negative charges allow $\mathrm{CNC}$ to bind to others positively charged molecules or polymeric systems. This is the case when cross-linking CNC with anionic molecules such as chitosan. CNC cross-linking with chitosan has been a subject of inquire for many authors [66-68] in the research of drug delivery as well as food packaging applications. Working under acid conditions, chitosan $\left(\mathrm{pK}_{\mathrm{a}}\right.$ of $\left.\sim 6.5\right)$ is positively charged [69] due to the protonation of its amino groups. Applications of chitosan are focused in fields such as drug delivery and based on its antimicrobial, haemostatic, wound healing and mucoadhesive properties [70]. Food packaging of chitosan are based on its biodegradable, biocompatible and strong antifungal activities [71-74].

Akhlaghi et al. [66] developed a novel drug delivery system based on a peptidic coupling reaction of oxidized $\mathrm{CNC}$ to graft chitosan oligosaccharide $\left(\mathrm{CO}_{\mathrm{OS}}\right)$. As a first step, hydroxyl groups of the surface of CNC were oxidized to carboxylic acid groups using TEMPO-mediated oxidation. Then, amino groups of $\mathrm{CO}_{\mathrm{OS}}$ reacted with carboxylic acid groups on oxidized $\mathrm{CNC}$ by action of EDC (1-ethyl-3-(3-dimethylaminopropyl) carbodiimide) and NHS (N-hydroxysuccinimide, 98\%+) cross-linker agents. Results revealed that modified $\mathrm{CNC}-\mathrm{g}-\mathrm{CO}_{\mathrm{OS}}$ showed a positive zeta potential due to the positive charges of $\mathrm{CO}_{\mathrm{OS}}$ in acid medium. In addition to a degree of substitution (DS) of 0.26 of carboxylate groups into amino groups. Previous results indicated that most of the carboxylic acid groups of the oxidized cellulose were involved in the peptidic reaction with $\mathrm{CO}_{\mathrm{OS}}$. 
Therefore, expanding outstanding improvement in polymeric applications can be employed by creating TEMPO-oxidized CNC to form a network with other bio-functional polymers.

\section{Non covalent surface modifications}

\section{Layer-by-layer}

Compared to covalent modification, the layer-by-layer technique consists in non-covalent hydrogen bonding and electrostatic interactions between the layers of positively charged polymers and negatively charged cellulose. This approach, which has been extended to materials such as proteins and colloids, relies on a consecutive adsorption of polyanions and polycations [75].

The advantage of having a layer-by-layer (LBL) assembly in food packaging is the addition film oxygen and moisture vapor barrier properties to the product [76,77], significant mechanical strength [78] as well as ultrathin and flexible film properties [77]. Limited research of $\mathrm{CNC}$ multilayer composite can be found in the area of food packaging, however recently de Mesquita et al. [67] developed a new biodegradable and biocompatible film combining anionic rod-like cellulose nanocrystals with cationic chitosan via LBL assembly technique. The sulfuric acid hydrolyzed cellulose nanocrystals and chitosan layer were applied onto a negatively charged glass or quartz slides. Subsequent immersion of the glass into the solutions of CNCs and chitosan was repeated until the desired quantities of bilayers were deposited. An intermediate step of rinsing was required in between each immersion in order to eliminate the material in excess. The authors concluded that a successful LBL assembly was produced, characterized by a thickness of $7.0 \mathrm{~nm}$ per single bilayer. Smooth surface and a dense and homogeneous distribution of nanocomposites in layers have been obtained.

Similar characteristics were found by the group Podsiadlo et al. [79] who reported a bilayer compound of cellulose nanocrystals with poly(diallyldimethylammonium chloride) of 11 $\mathrm{nm}$ thickness. Surface morphology was characterized by atomic force microscopy (AFM) and scanning electron microscopy (SEM). Results revealed a uniform coverage and tightly packed cellulose nanocrystals layers.

Formation with other cationic polymers with convenient properties for food packaging can also be achieved by this method. It is interesting to note that, because of the negatively charged nature of CNC and its good packing capacity with other polymers not only mechanical properties can be enhanced, but also functional characteristics.

\section{Cationic surfactant interaction}

An important characteristic of CNC, prepared under sulphuric oxidation, is that it becomes negatively charged due to the sulphate ester remained group of the acid treatment [80]. Aloulou et al. 2004 found by zeta potential method that anionic charges of cellulose are around $-10 \mathrm{mV}$. In this context, positive charges from cationic surfactant can be adsorbed onto the negatively charged cellulose surface with the aim of adding hydrophobic 
404

405

406

407

408

409

410

411

412

413

414

415

416

417

418

419

420

421

422

423

424

425

426

427

428

429

430

431

432

433

434

435

436

437

438

439

440

441

442

443

444

445

446

447

448 properties. Applications of cationic surfactants have been focused in areas such as organic pollutants and toxic substances removal [81], drug delivery systems [82] and surface modification using admicellar polymerization [83].

A surfactant, is a molecule which consists of a polar head, soluble in water and hydrophobic alkyl chain, insoluble in water. His amphiphilic behavior act by reducing the surface tension between two non-miscible components. Some cationic polymers are used in food packaging because of their antimicrobial activities. The most potent antimicrobial agent, highly used for active food packaging materials because of its tasteless and odorless properties according to the article 3 of European Regulation [84] is lauric-arginate (LAE). Lauric-arginate or also called $\mathrm{N}^{\alpha}$-lauroyl-arginine ethyl ester monohydrochloride, is an cationic amino-acid based surfactant that is derivative of lauric acid, L-arginine and ethanol $[85,86]$. LAE has the property to extend the shelf life of milk products by controlling bacterial growth [87], as well as an efficient action on the cytoplasmic membranes of the microorganisms which leads to alter their metabolic process $[85,86,89]$. Due to the fact that LAE is quickly metabolized within the human body and prevent microbial growth in food products, its application make it valuable for food products [90].

Hence, bioactive films were proposed by Muriel-Galet et al. [88] where LAE reinforced ethylene-vinyl alcohol (EVOH) film, showed transparent and optical properties and good antimicrobial release $(80 \%)$ at $23^{\circ} \mathrm{C}$ when $5 \%$ and $10 \%$ LAE were added in EVOH polymer.

Studies made by Asker et al. [90] suggested that cationic surfactants mixed with non-ionic surfactants creates micelles with anionic polysaccharides. The research group indicated that antimicrobial activity of LAE in combination with non-ionic Tween 20 (T20) leads to a stable solution when pectin was used as anionic polysaccharide. Due to the fact that food can also be stored at ambient or cold temperatures, this parameter was of great importance. Resulted LAE/T20 micelles loaded in pectin based suspensions were more stable to aggregation in temperatures of $4{ }^{\circ} \mathrm{C}$ and $22^{\circ} \mathrm{C}$ during a period of time of $1-2$ weeks than suspensions in the absence of T20 [90].

LAE micelles systems chitosan-based films have been tested on the surface of fresh chicken breast fillets. An antimicrobial effect was found along with a significant decrease of growth reduction (> $4 \mathrm{log}$ ) for mesophiles, psychrophiles and Pseudomonas spp. yeast and fungi. Chitosan films evidenced an antimicrobial effect in the range 0.47-2.96 log reductions, while chitosan-5\%LAE film produced 1.78-5.81 log reduction. Similar results were obtained against coliform bacteria and hydrogen sulfide-producing bacteria [91].

These results may indicate that anionic biopolymers can have an antimicrobial activity by adding cationic surfactants in their matrices. Other amino-acid based surfactants such as the arginine-based cationic $\mathrm{N}^{\alpha}$-acyl-arginine-methyl ester hydrochloride, arginine- $\mathrm{N}$-alkyl amide dihydrochloride and arginine-O-alkyl ester dihydrochloride can also be used, because of their non-toxicity and biodegradability in combination with antimicrobial properties [92]. 
Similar results can be expected of $\mathrm{CNC}$ when a cationic surfactant, such as LAE, interacts with its negative charges. Figure 3 shows a schematic procedure of ionic interaction between LAE and CNC.

\section{Radiation induced graft copolymerization}

Modification of polymers surface by grafting monomers onto active sites has been an attractive method to give additional functionalities to the polymer backbone. Surface grafting polymerization is, in most of the cases, induced by decomposition of a chemical initiator which propagates the reaction, however the use of other initiators such as ozone [93], $\gamma$-rays [94], electron beam [95], plasma [96], corona discharge [97] and ultraviolet irradiation [98] have also been employed. In radiation-induced graft copolymerization method, active sites are produced on the polymer backbone using high energy radiation, thus, the irradiated polymer can react with monomer units, which propagate to form side chain grafts [99]. Radiation-induced graft polymerization have the advantages of its simplified process, no residual by-products and low cost of production [100]. It also offers the possibility of initiating the polymerization in a wide range of temperatures and under various experimental conditions such as bulk, solution, and emulsion or solid [101].

\section{Method of radiation-induced graft polymerization}

Two methods are involved in radiation-induced graft copolymerization: the first method presented is called simultaneous irradiation where a polymer in the presence of a monomer are activated together to form free radicals from both polymer backbone and monomer units. Thus, monomer is immediately grafted to polymer backbone and polymerization is started. This type of irradiation can be carried out in air, under inert atmosphere (e.g. $\mathrm{N}_{2}$ ) or vacuum [99]; the reaction mechanism is presented in the following equations (eqs. 1-4).
Irradiation:
$P \stackrel{\gamma-\text { rays }}{\longrightarrow} P$
Initiation:
$P^{*}+M \rightarrow P M$
(primary radicals)
(graft chain)
Propagation:
$P M+n M \rightarrow P M_{n+1}$
(graft growing chains)
Termination:
$P M_{n}+P M_{m} \rightarrow P M_{n+m}$
(graft polymer)

The second method is called pre-irradiation where the polymer is irradiated in the absence of the monomer, followed by immersion in the monomer solution [99,102]. If the irradiation step is carried out in air, the generated radical react with oxygen to form peroxides and hydroperoxides, thus, polymerization is finished. However, this effect is reversible when thermal degradation of hydroperoxides takes place, thus, polymerization can be re-activated. On the other hand, when irradiation is performed in the absence of air, the irradiated polymer created radicals that remain trapped on the polymer backbone and initiate grafting in the presence of monomer units as explained previously.

Limitations of this technique are the high levels of production of monomer radical rather than growing chains of polymer radicals, leading to a non-controlled method. To overcome this problem, many studies $[62,103]$ have presented a controlled RAFT polymerization with irradiation. 
In a recent study, poly(hydroxyethylmethacrylate) was grafted from surface with a RAFT agent (cumyldithiobenzoate, CDB) by gamma-irradiation at $5.98 \mathrm{kGy}$ [104]. The resulted cellulose- $g$-PHEMA showed a controlled grafting of HEMA monomer while changing the $[\mathrm{HEMA}] /[\mathrm{CDB}]$ ratio. Compared to the polydispersity (PD) achieved by conventional grafting technique (19.6), RAFT-mediated polymerization PD was 2.5. An increase of hydrophobicity due to the grafted PHEMA was observed from contact angle measurements. The authors observed that by increasing the degree of grafting of PHEMA to cellulose from 11 to $44.5 \%$, the contact angle increases from $18.2^{\circ}$ to $55.4^{\circ}$. Barsbay et al. [103] also reported effective results after polystyrene grating onto cellulose via radiation-induced polymerization. Enzymatic stability of cellulose- $g$-polystyrene with $39 \%$ graft ratio was proven after 3 weeks of testing against Trichoderma reesei hydrolysis, compared to the rapid degradation seen in non-modified cellulose. The resulting cellulosic materials showed a complete protection against the enzymatic attack, indicating an efficient polystyrene covering onto the surface of the cellulose.

Antibacterial activity was also improved onto cotton fabric after radiation-induced grafting of vinylbenzyltrimethylammonium chloride (VBT) [105]. It was suggested that increasing the irradiation dose from 2 to $8 \mathrm{kGy}$ the grafting yield of VBT onto cellulose increases. By working with a grafting yield $\sim 25 \%$ of VBT onto cotton cellulose substrate showed an approximately $6 \mathrm{log}$ cycle reduction in bacterial counts of Escherichia coli and Staphylococcus aureus with respect to the control sample within $6 \mathrm{~h}$ of exposure. This application done on antibacterial cotton tissues was analyzed before and after washing with commercial detergent powder, demonstrating that the antibacterial activity for both microorganisms was not affected after 4 washing cycles .

Lacroix et al. [106] who found that grafting polymers via gamma irradiation enhances the interaction within polymer blends, the film formation and interfacial adhesion of multilayered systems, resulting in improved mechanical properties. In this study, zein and poly(vinyl alcohol) (PVA) were gamma-irradiated in the presence of different ratios of acrylic acid (AAc) monomer. The grafted films (zein/PVA-g-AAc) showed an improvement of puncture strength (PS) and puncture deformation (PD) of $30 \%$ and $50 \%$, respectively by adding to PVA $5 \%$ of monomer under $20 \mathrm{kGy}$. Similar behaviors were observed on grafted $35 \%$ of 2-hydroxyethylmethacrylate (HEMA) or silane in methylcellulose under $10 \mathrm{kGy}$. Mechanical properties improvements were reported with values of PS of 282-296 N.mm ${ }^{-1}$ and PD of 5.0-5.5 mm, as compared to $147 \mathrm{~N} . \mathrm{mm}^{-1}$ and $3.96 \mathrm{~mm}$ respectively for ungrafted films. Finally, a trilayer grafted composite film formed by binding polycaprolactone (PCL)/chitosan with silane-grafted chitosan under $10 \mathrm{kGy}$ showed a higher tensile strength of $22 \mathrm{MPa}$, because of the interlayer adhesion of molecules. The use of CNC as a reinforcing agent and trimethylolpropane trimethylacrylate (TMPTMA) as grafted plasticizer in methylcellulose-based irradiated films creates a tortuosity and decreases the water vapor permeability (WVP) in the films of 25\% [107].

According to these studies, either mechanical, physicochemical or antimicrobial properties can be improved after using graft-polymerization via gamma-irradiation. Development in new biodegradable materials can focus in this relevant method for including to their systems bioactive monomers in packaging sectors. 


\section{Conclusions and outlook}

This review has provided an overview of the emerging modifications of CNC surface for bioactive food packaging applications. Taking advantage of CNC surface functional groups, reactions such as acetylation, polymer grafting, TEMPO-mediated oxidation or radiation-induced polymerization can be applied. Thus, a more stable, hydrophobic and active cellulose can be expected depending on the procedure used.

Acetylation was shown to improve the hydrophobicity of cellulose surface, leading to a better compatibility with non-polar active molecules or polymer matrices. Only when heterogeneous acetylation is employed, morphological changes may occur due to the acetate cellulose fibers that are stripped and dissolved into the reactive medium. Polymerization either in situ or by pre-synthesized can be produced via polymer grafting techniques, controlled grafting polymerization and a narrow polydispersity on cellulose surface is achieved when RAFT polymerization is carried out.

Negative charges introduced onto cellulose surface will play an important role when cationic polymers are added. Polyelectrolyte interactions can be induced by TEMPOmediated oxidation or layer-by-layer assembly.

Due to their improved and novel compatibility of modified CNC with various organic or inorganic compounds, these conjugates could become material interesting in many others areas such as engineering and medical fields. It is important to underline that the desired functional compound to be attached requires a previous study in order to observe the efficacy of the final product.

\section{Aknowledgements}

This research was supported by the National Science and Engineering Research Council of Canada (NSERC) and FPInnovations (Pointe-Claire, Canada) through the RDC program. 


\section{References}

593 [1] Theron MM, Lues JFR. Organic Acids and Meat Preservation: A Review. Food Rev Int

601

602

603

604

605

606

607

608

609

610

611

612

613

614

615 2007;23:141-58.

[2] Lopez-Rubio A, Gavara R, Lagaron JM. Bioactive packaging: turning foods into healthier foods through biomaterials. Trends Food Sci Technol 2006;17:567-75.

[3] Röhr A, Lüddecke K, Drusch S, Müller MJ, Alvensleben R v. Food quality and safetyconsumer perception and public health concern. Food Control 2005;16:649-55.

[4] Khan A, Huq T, Khan RA, Riedl B, Lacroix M. Nanocellulose-Based Composites and Bioactive Agents for Food Packaging. Crit Rev Food Sci Nutr 2014;54:163-74.

[5] Azapagic A, Emsley A, Hamerton I. Polymers: The Environment and Sustainable Development. John Wiley \& Sons; 2003.

[6] Leceta I, Etxabide A, Cabezudo S, de la Caba K, Guerrero P. Bio-based films prepared with by-products and wastes: environmental assessment. J Clean Prod 2014;64:218-27.

[7] Appendini P, Hotchkiss JH. Review of antimicrobial food packaging. Innov Food Sci Emerg Technol 2002;3:113-26.

[8] Bautista-Baños S, Hernández-Lauzardo AN, Velázquez-del Valle MG, Hernández-López M, Ait Barka E, Bosquez-Molina E, et al. Chitosan as a potential natural compound to control pre and postharvest diseases of horticultural commodities. Crop Prot 2006;25:108-18.

[9] Zivanovic S, Chi S, Draughon AF. Antimicrobial Activity of Chitosan Films Enriched with Essential Oils. J Food Sci 2005;70:M45-M51.

[10] Azeredo HMC de. Nanocomposites for food packaging applications. Food Res Int 2009;42:1240-53.

[11] Suyatma NE, Tighzert L, Copinet A, Coma V. Effects of Hydrophilic Plasticizers on Mechanical, Thermal, and Surface Properties of Chitosan Films. J Agric Food Chem 2005;53:3950-7.

[12] Ludueña LN, Alvarez VA, Vazquez A. Processing and microstructure of $\mathrm{PCL} / \mathrm{clay}$ nanocomposites. Mater Sci Eng A 2007;460-461:121-9.

[13] Rhim J-W, Park H-M, Ha C-S. Bio-nanocomposites for food packaging applications. Prog Polym Sci 2013;38:1629-52.

[14] Klemm D, Schumann D, Kramer F, Heßler N, Koth D, Sultanova B. Nanocellulose Materials Different Cellulose, Different Functionality. Macromol Symp 2009;280:60-71.

[15] Azeredo HMC de. Nanocomposites for food packaging applications. Food Res Int 2009;42:1240-53.

[16] Sun D, Zhou L, Wu Q, Yang S. Preliminary research on structure and properties of nanocellulose. J Wuhan Univ Technol-Mater Sci Ed 2007;22:677-80.

[17] Ray S, Quek SY, Easteal A, Chen XD. The Potential Use of Polymer-Clay Nanocomposites in Food Packaging. Int J Food Eng 2006;2.

[18] Alexandre M, Dubois P. Polymer-layered silicate nanocomposites: preparation, properties and uses of a new class of materials. Mater Sci Eng R Rep 2000;28:1-63.

[19] Erdohan ZÖ, Turhan KN. Barrier and mechanical properties of methylcellulose-whey protein films. Packag Technol Sci 2005;18:295-302. 
633 [20] Ye D, Farriol X. Factors influencing molecular weights of methylcelluloses prepared from annual plants and juvenile eucalyptus. J Appl Polym Sci 2006;100:1785-93.

[21] Shih C-M, Shieh Y-T, Twu Y-K. Preparation and characterization of cellulose/chitosan blend films. Carbohydr Polym 2009;78:169-74.

[22] Dufresne A. Nanocellulose: From Nature to High Performance Tailored Materials. Walter de Gruyter; 2012.

[23] Sjöström E. Wood Chemistry: Fundamentals and Applications. Gulf Professional Publishing; 1993.

640

641

[24] Azizi Samir MAS, Alloin F, Dufresne A. Review of recent research into cellulosic whiskers, their properties and their application in nanocomposite field. Biomacromolecules 2005;6:612-26.

[25] Habibi Y, Lucia LA, Rojas OJ. Cellulose nanocrystals: chemistry, self-assembly, and applications. Chem Rev 2010;110:3479-500.

[26] Rånby BG, Banderet A, Sillén LG. Aqueous Colloidal Solutions of Cellulose Micelles. Acta Chem Scand 1949;3:649-50.

[27] Hamad W. On the Development and Applications of Cellulosic Nanofibrillar and Nanocrystalline Materials. Can J Chem Eng 2006;84:513-9.

[28] Letchford, Jackson, Wasserman B, Ye, Hamad W, Burt H. The use of nanocrystalline cellulose for the binding and controlled release of drugs. Int J Nanomedicine 2011:321.

[29] De Nooy A e. j., Besemer A c., van Bekkum H. Highly selective tempo mediated oxidation of primary alcohol groups in polysaccharides. Recl Trav Chim Pays-Bas 1994;113:165-6.

[30] Habibi Y, Chanzy H, Vignon MR. TEMPO-mediated surface oxidation of cellulose whiskers. Cellulose 2006;13:679-87.

[31] Da Silva Perez D, Montanari S, Vignon MR. TEMPO-Mediated Oxidation of Cellulose III. Biomacromolecules 2003;4:1417-25.

[32] Ouattara B, Sabato SF, Lacroix M. Combined effect of antimicrobial coating and gamma irradiation on shelf life extension of pre-cooked shrimp (Penaeus spp.). Int J Food Microbiol 2001;68:1-9.

[33] Oussalah M, Caillet S, Salmiéri S, Saucier L, Lacroix M. Antimicrobial effects of alginatebased films containing essential oils on Listeria monocytogenes and Salmonella typhimurium present in bologna and ham. J Food Prot 2007;70:901-8.

[34] Yamanaka S, Sugiyama J. Structural modification of bacterial. Cellulose 2000;7:213-25.

[35] Ghosh K, Srivatsa A, Nirmala N, Sharma T. Development and Application of Fungistatic Wrappers in Food Preservation .2. Wrappers Made by Coating Process. J Food Sci TechnolMysore 1977;14:261-4.

[36] Han JH, Floros JD. Casting antimicrobial packaging films and measuring their physical properties and antimicrobial activity. J Plast Film Sheeting 1997;13:287-98.

[37] Curcio M, Puoci F, lemma F, Parisi OI, Cirillo G, Spizzirri UG, et al. Covalent insertion of antioxidant molecules on chitosan by a free radical grafting procedure. J Agric Food Chem 2009;57:5933-8.

[38] Spizzirri UG, lemma F, Puoci F, Cirillo G, Curcio M, Parisi Ol, et al. Synthesis of antioxidant polymers by grafting of gallic acid and catechin on gelatin. Biomacromolecules 2009;10:1923-30.

[39] Spizzirri UG, Parisi OI, lemma F, Cirillo G, Puoci F, Curcio M, et al. Antioxidantpolysaccharide conjugates for food application by eco-friendly grafting procedure. Carbohydr Polym 2010;79:333-40.

[40] Seifried HE, Anderson DE, Fisher El, Milner JA. A review of the interaction among dietary antioxidants and reactive oxygen species. J Nutr Biochem 2007;18:567-79. 
701

702

703

704

705

706

707

708

709

710

711

712

713

714

715

716

717

718

719

720

721

722

723

724

725

726

727

728

[41] Valko M, Leibfritz D, Moncol J, Cronin MTD, Mazur M, Telser J. Free radicals and antioxidants in normal physiological functions and human disease. Int J Biochem Cell Biol 2007;39:44-84.

[42] Liu J, Luo J, Ye H, Zeng X. Preparation, antioxidant and antitumor activities in vitro of different derivatives of levan from endophytic bacterium Paenibacillus polymyxa EJS-3. Food Chem Toxicol 2012;50:767-72.

[43] Jin M, Lu Z, Huang M, Wang Y, Wang Y. Sulfated modification and antioxidant activity of exopolysaccahrides produced by Enterobacter cloacae Z0206. Int J Biol Macromol 2011;48:607-12.

[44] Jonoobi M, Harun J, Mathew AP, Hussein MZB, Oksman K. Preparation of cellulose nanofibers with hydrophobic surface characteristics. Cellulose 2010;17:299-307.

[45] Sassi J-F, Chanzy H. Ultrastructural aspects of the acetylation of. Cellulose 1995;2:111-27.

[46] Buras EM, Hobart SR, Hamalainen C, Cooper AS. A- Preliminary Report on Fully Acetylated Cotton. Text Res J 1957;27:214-22.

[47] Braun B, Dorgan JR. Single-Step Method for the Isolation and Surface Functionalization of Cellulosic Nanowhiskers. Biomacromolecules 2009;10:334-41.

[48] Zhao B, Brittain WJ. Polymer brushes: surface-immobilized macromolecules. Prog Polym Sci 2000;25:677-710.

[49] Masuda T, Inaba $Y$, Maekawa T, Takeda $Y$, Yamaguchi $H$, Nakamoto $K$, et al. Simple detection method of powerful antiradical compounds in the raw extract of plants and its application for the identification of antiradical plant constituents. J Agric Food Chem 2003;51:1831-8.

[50] Ardestani A, Yazdanparast R. Antioxidant and free radical scavenging potential of Achillea santolina extracts. Food Chem 2007;104:21-9.

[51] Ljungberg N, Bonini C, Bortolussi F, Boisson C, Heux L, Cavaillé JY. New nanocomposite materials reinforced with cellulose whiskers in atactic polypropylene: effect of surface and dispersion characteristics. Biomacromolecules 2005;6:2732-9.

[52] Cao X, Chen Y, Chang PR, Stumborg M, Huneault MA. Green composites reinforced with hemp nanocrystals in plasticized starch. J Appl Polym Sci 2008;109:3804-10.

[53] Roy D, Knapp JS, Guthrie JT, Perrier S. Antibacterial cellulose fiber via RAFT surface graft polymerization. Biomacromolecules 2008;9:91-9.

[54] Gabrielska J, Sarapuk J, Przestalski S. Investigations of new bis-ammonium salts with potential biological application. Tenside Surfactants Deterg n.d.;31:296-8.

[55] Block SS. Disinfection, Sterilization, and Preservation. Lippincott Williams \& Wilkins; 2001.

[56] FRANKLIN TJ, SNOW GA. Biochemistry of antimicrobial action. 3rd edition. 1981:xi +217 pp.

[57] Kanazawa A, Ikeda T, Endo T. Novel polycationic biocides: Synthesis and antibacterial activity of polymeric phosphonium salts. J Polym Sci Part Polym Chem 1993;31:335-43.

[58] Kanazawa A, Ikeda T, Endo T. Polymeric phosphonium salts as a novel class of cationic biocides. VII. Synthesis and antibacterial activity of polymeric phosphonium salts and their model compounds containing long alkyl chains. J Appl Polym Sci 1994;53:1237-44.

[59] Dizman B, Elasri MO, Mathias LJ. Synthesis and antibacterial activities of water-soluble methacrylate polymers containing quaternary ammonium compounds. J Polym Sci Part Polym Chem 2006;44:5965-73.

[60] Ignatova M, Voccia S, Gilbert B, Markova N, Mercuri PS, Galleni M, et al. Synthesis of copolymer brushes endowed with adhesion to stainless steel surfaces and antibacterial properties by controlled nitroxide-mediated radical polymerization. Langmuir ACS J Surf Colloids 2004;20:10718-26. 
[61] Venkataraman S, Zhang Y, Liu L, Yang Y-Y. Design, syntheses and evaluation of hemocompatible pegylated-antimicrobial polymers with well-controlled molecular structures. Biomaterials 2010;31:1751-6.

[62] Millard P-E, Barner L, Stenzel MH, Davis TP, Barner-Kowollik C, Müller AHE. RAFT Polymerization of $\mathrm{N}$-Isopropylacrylamide and Acrylic Acid under $\mathrm{y}$-Irradiation in Aqueous Media. Macromol Rapid Commun 2006;27:821-8.

[63] Quinn JF, Barner L, Rizzardo E, Davis TP. Living free-radical polymerization of styrene under a constant source of $\gamma$ radiation. J Polym Sci Part Polym Chem 2002;40:19-25.

[64] Montanari S, Roumani M, Heux L, Vignon MR. Topochemistry of Carboxylated Cellulose Nanocrystals Resulting from TEMPO-Mediated Oxidation. Macromolecules 2005;38:166571.

[65] Araki J, Wada M, Kuga S. Steric Stabilization of a Cellulose Microcrystal Suspension by Poly(ethylene glycol) Grafting. Langmuir 2001;17:21-7.

[66] Akhlaghi SP, Berry RC, Tam KC. Surface modification of cellulose nanocrystal with chitosan oligosaccharide for drug delivery applications. Cellulose 2013;20:1747-64.

[67] De Mesquita JP, Donnici CL, Pereira FV. Biobased Nanocomposites from Layer-by-Layer Assembly of Cellulose Nanowhiskers with Chitosan. Biomacromolecules 2010;11:473-80.

[68] Wang H, Roman M. Formation and Properties of Chitosan-Cellulose Nanocrystal Polyelectrolyte-Macroion Complexes for Drug Delivery Applications. Biomacromolecules 2011;12:1585-93.

[69] Ogawa S, Decker EA, McClements DJ. Production and characterization of O/W emulsions containing droplets stabilized by lecithin-chitosan-pectin mutilayered membranes 2004:3595-600.

[70] Sonia TA, Sharma CP. Chitosan and Its Derivatives for Drug Delivery Perspective. In: Jayakumar R, Prabaharan M, Muzzarelli RAA, editors. Chitosan Biomater. I, Springer Berlin Heidelberg; 2011, p. 23-53.

[71] Darmadji P, Izumimoto M. Effect of chitosan in meat preservation. Meat Sci 1994;38:24354.

[72] Kim KW, Min BJ, Kim Y-T, Kimmel RM, Cooksey K, Park SI. Antimicrobial activity against foodborne pathogens of chitosan biopolymer films of different molecular weights. LWT Food Sci Technol 2011;44:565-9.

[73] No HK, Meyers SP, Prinyawiwatkul W, Xu Z. Applications of chitosan for improvement of quality and shelf life of foods: a review. J Food Sci 2007;72:R87-100.

[74] Rabea El, Badawy ME-T, Stevens CV, Smagghe G, Steurbaut W. Chitosan as Antimicrobial Agent: Applications and Mode of Action. Biomacromolecules 2003;4:1457-65.

[75] Decher G. Fuzzy Nanoassemblies: Toward Layered Polymeric Multicomposites. Science 1997;277:1232-7.

[76] Jang W-S, Rawson I, Grunlan JC. Layer-by-layer assembly of thin film oxygen barrier. Thin Solid Films 2008;516:4819-25.

[77] Duncan TV. Applications of nanotechnology in food packaging and food safety: Barrier materials, antimicrobials and sensors. J Colloid Interface Sci 2011;363:1-24.

[78] Chen W, McCarthy TJ. Layer-by-Layer Deposition: A Tool for Polymer Surface Modification. Macromolecules 1997;30:78-86.

[79] Podsiadlo P, Choi S-Y, Shim B, Lee J, Cuddihy M, Kotov NA. Molecularly Engineered Nanocomposites: Layer-by-Layer Assembly of Cellulose Nanocrystals. Biomacromolecules 2005;6:2914-8.

[80] Peng BL, Dhar N, Liu HL, Tam KC. Chemistry and applications of nanocrystalline cellulose and its derivatives: A nanotechnology perspective. Can J Chem Eng 2011;89:1191-206. 
[81] Nayyar SP, Sabatini DA, Harwell JH. Surfactant Adsolubilization and Modified Admicellar Sorption of Nonpolar, Polar, and Ionizable Organic Contaminants. Environ Sci Technol 1994;28:1874-81.

[82] Hayakawa K, Mouri Y, Maeda T, Satake I, Sato M. Surfactant-modified zeolites as a drug carrier and the release of chloroquin. Colloid Polym Sci 2000;278:553-8.

[83] Boufi S, Gandini A. Formation of polymeric films on cellulosic surfaces by admicellar polymerization. Cellulose 2001;8:303-12.

[84] EUR-Lex - Official Journal n.d.

[85] Infante MR, Pérez L, Pinazo A, Clapés $P$, Morán MC, Angelet $M$, et al. Amino acid-based surfactants. Comptes Rendus Chim 2004;7:583-92.

[86] Ruckman SA, Rocabayera X, Borzelleca JF, Sandusky CB. Toxicological and metabolic investigations of the safety of $\mathrm{N}$ - $\alpha$-Lauroyl-I-arginine ethyl ester monohydrochloride (LAE). Food Chem Toxicol 2004;42:245-59.

[87] Woodcock NH, Hammond BH, Ralyea RD, Boor KJ. Short communication: Na-Lauroyl-Iarginine ethylester monohydrochloride reduces bacterial growth in pasteurized milk. J Dairy Sci 2009;92:4207-10.

[88] Muriel-Galet V, López-Carballo G, Hernández-Muñoz P, Gavara R. Characterization of ethylene-vinyl alcohol copolymer containing lauril arginate (LAE) as material for active antimicrobial food packaging. Food Packag Shelf Life 2014;1:10-8.

[89] Infante $M$, Pinazo A, Seguer J. Non-conventional surfactants from amino acids and glycolipids: Structure, preparation and properties. Colloids Surf Physicochem Eng Asp 1997;123-124:49-70.

[90] Asker D, Weiss J, McClements DJ. Formation and stabilization of antimicrobial delivery systems based on electrostatic complexes of cationic-non-ionic mixed micelles and anionic polysaccharides. J Agric Food Chem 2011;59:1041-9.

[91] Higueras L, López-Carballo G, Hernández-Muñoz P, Gavara R, Rollini M. Development of a novel antimicrobial film based on chitosan with LAE (ethyl-N( $\alpha$ )-dodecanoyl-I-arginate) and its application to fresh chicken. Int J Food Microbiol 2013;165:339-45.

[92] Clapés P, Rosa Infante M. Amino Acid-based Surfactants: Enzymatic Synthesis, Properties and Potential Applications. Biocatal Biotransformation 2002;20:215-33.

[93] Partouche E, Waysbort D, Margel S. Surface modification of crosslinked poly(styrenedivinyl benzene) micrometer-sized particles of narrow size distribution by ozonolysis. J Colloid Interface Sci 2006;294:69-78.

[94] Bucio E, Skewes P, Burillo G. Synthesis and characterization of azo acrylates grafted onto polyethylene terephthalate by gamma irradiation. Nucl Instrum Methods Phys Res Sect B Beam Interact Mater At 2005;236:301-6.

[95] Vahdat A, Bahrami H, Ansari N, Ziaie F. Radiation grafting of styrene onto polypropylene fibres by a $10 \mathrm{MeV}$ electron beam. Radiat Phys Chem 2007;76:787-93.

[96] Okubo M, Tahara M, Saeki N, Yamamoto T. Surface modification of fluorocarbon polymer films for improved adhesion using atmospheric-pressure nonthermal plasma graftpolymerization. Thin Solid Films 2008;516:6592-7.

[97] Lei J, Shi M, Zhang J. Surface graft copolymerization of hydrogen silicone fluid onto fabric through corona discharge and water repellency of grafted fabric. Eur Polym J 2000;36:1277-81.

[98] Stannett VT. Radiation grafting - State-of-the-art. Radiat Phys Chem 1990;35:82-7.

[99] Nasef MM, Hegazy E-SA. Preparation and applications of ion exchange membranes by radiation-induced graft copolymerization of polar monomers onto non-polar films. Prog Polym Sci 2004;29:499-561. 
825

826

827

828

829

830

831

832

833

834

835

836

837

838

839

840

841

842

843

844

845

846

847

848

849

850

851

852

853

854

855

856

857

858

859

860

861

862

863

864

865

866

867

868

869

870

871

[100] Cheremisinoff P. Handbook of Engineering Polymeric Materials. CRC Press; 1997.

[101] Chapiro A. Radiation induced grafting. Radiat Phys Chem 1977;9:55-67.

[102] Kobayashi Y. Gamma-ray-induced graft copolymerization of styrene onto cellulose and some chemical properties of the grafted polymer. J Polym Sci 1961;51:359-72.

[103] Barsbay M, Güven O, Stenzel MH, Davis TP, Barner-Kowollik C, Barner L. Verification of Controlled Grafting of Styrene from Cellulose via Radiation-Induced RAFT Polymerization. Macromolecules 2007;40:7140-7.

[104] Kodama Y, Barsbay M, Güven O. Radiation-induced and RAFT-mediated grafting of poly(hydroxyethyl methacrylate) (PHEMA) from cellulose surfaces. Radiat Phys Chem 2014;94:98-104.

[105] Kumar V, Bhardwaj YK, Rawat KP, Sabharwal S. Radiation-induced grafting of vinylbenzyltrimethylammonium chloride (VBT) onto cotton fabric and study of its antibacterial activities. Radiat Phys Chem 2005;73:175-82.

[106] Lacroix M, Khan R, Senna M, Sharmin N, Salmieri S, Safrany A. Radiation grafting on natural films. Radiat Phys Chem 2014;94:88-92.

[107] Khan RA, Salmieri S, Dussault D, Uribe-Calderon J, Kamal MR, Safrany A, et al. Production and Properties of Nanocellulose-Reinforced Methylcellulose-Based Biodegradable Films. J Agric Food Chem 2010;58:7878-85. 


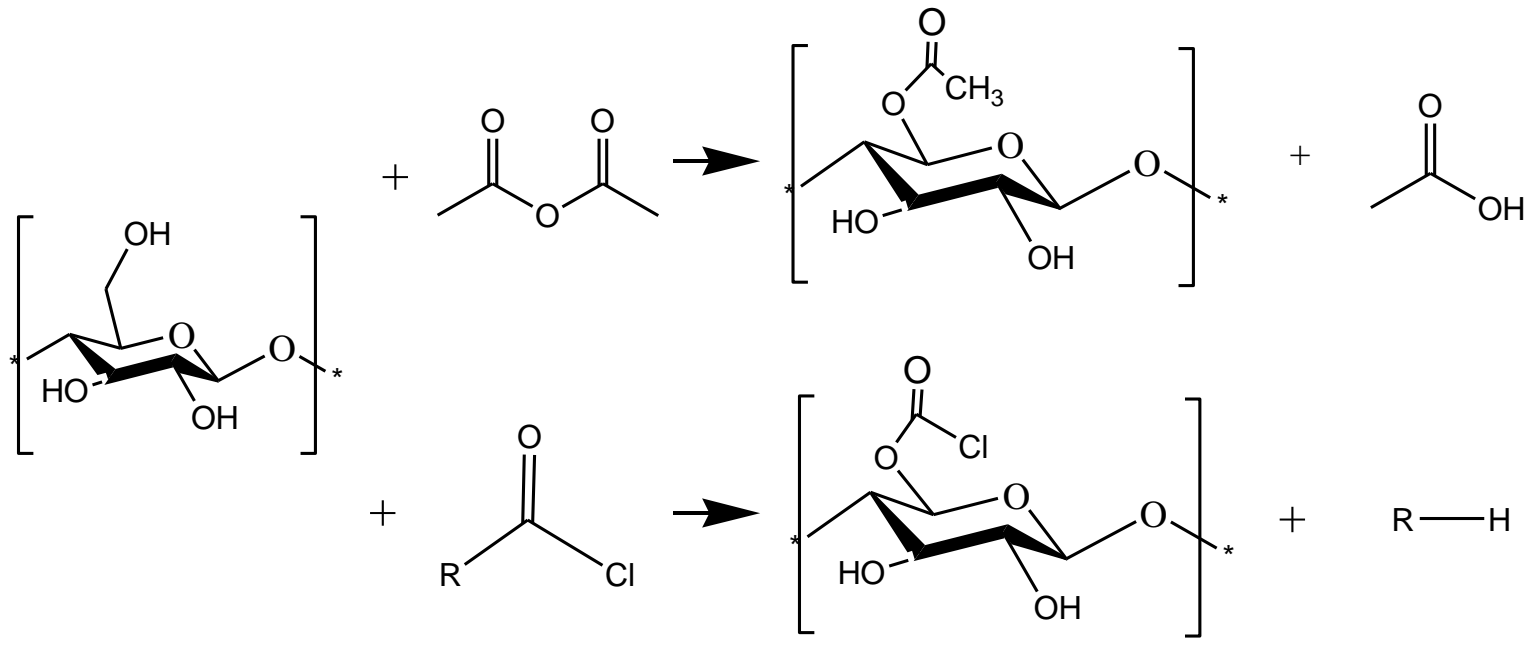

874

875

876

877

878

879

880

881

882

883

884

885

886

887

888

889

890

891

892

893

894

895

896

897
Fig. 1. The proposed mechanism of reaction of $\mathrm{CNC}$ with acetic anhydride and acetyl chloride reagents. 

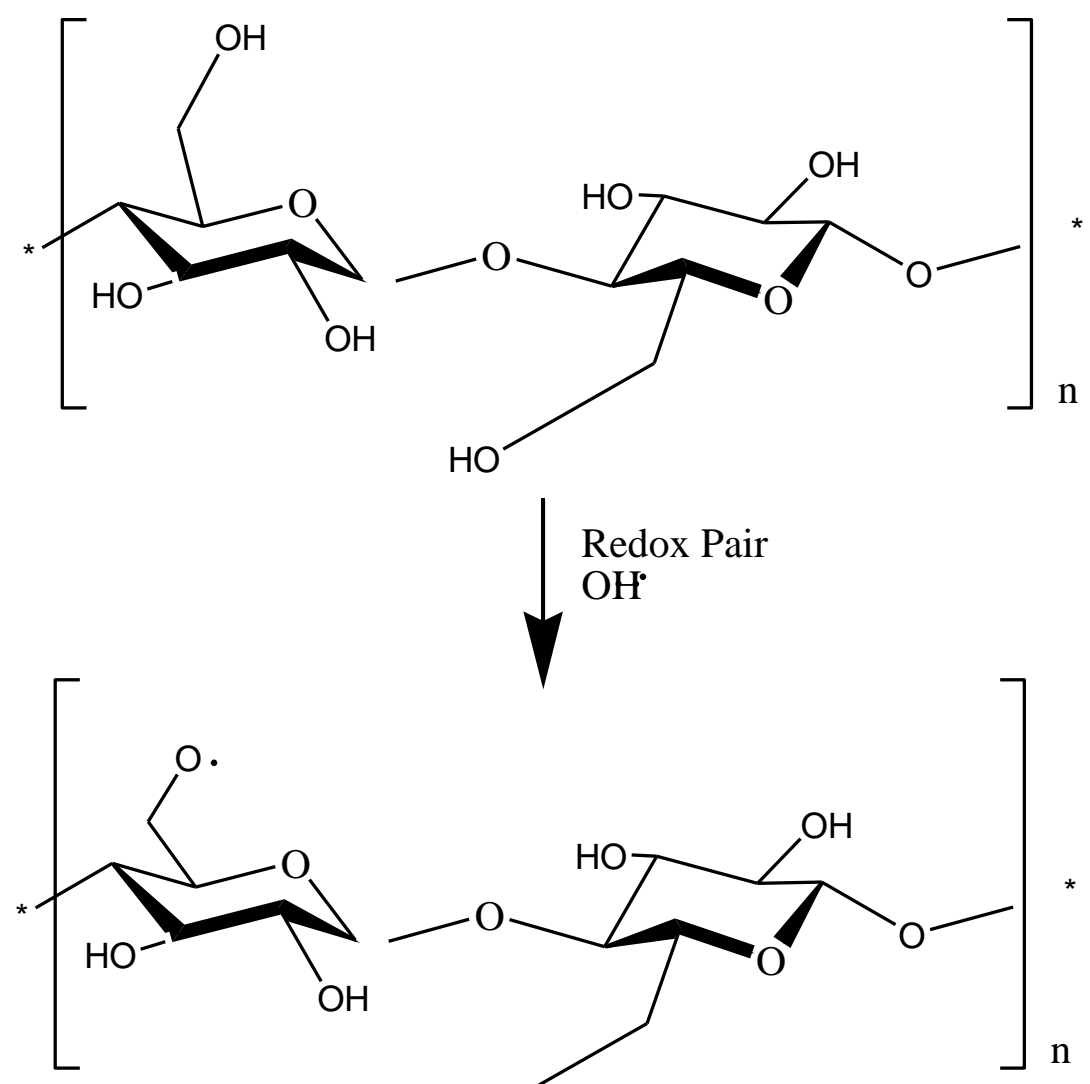

CNC macroradicals
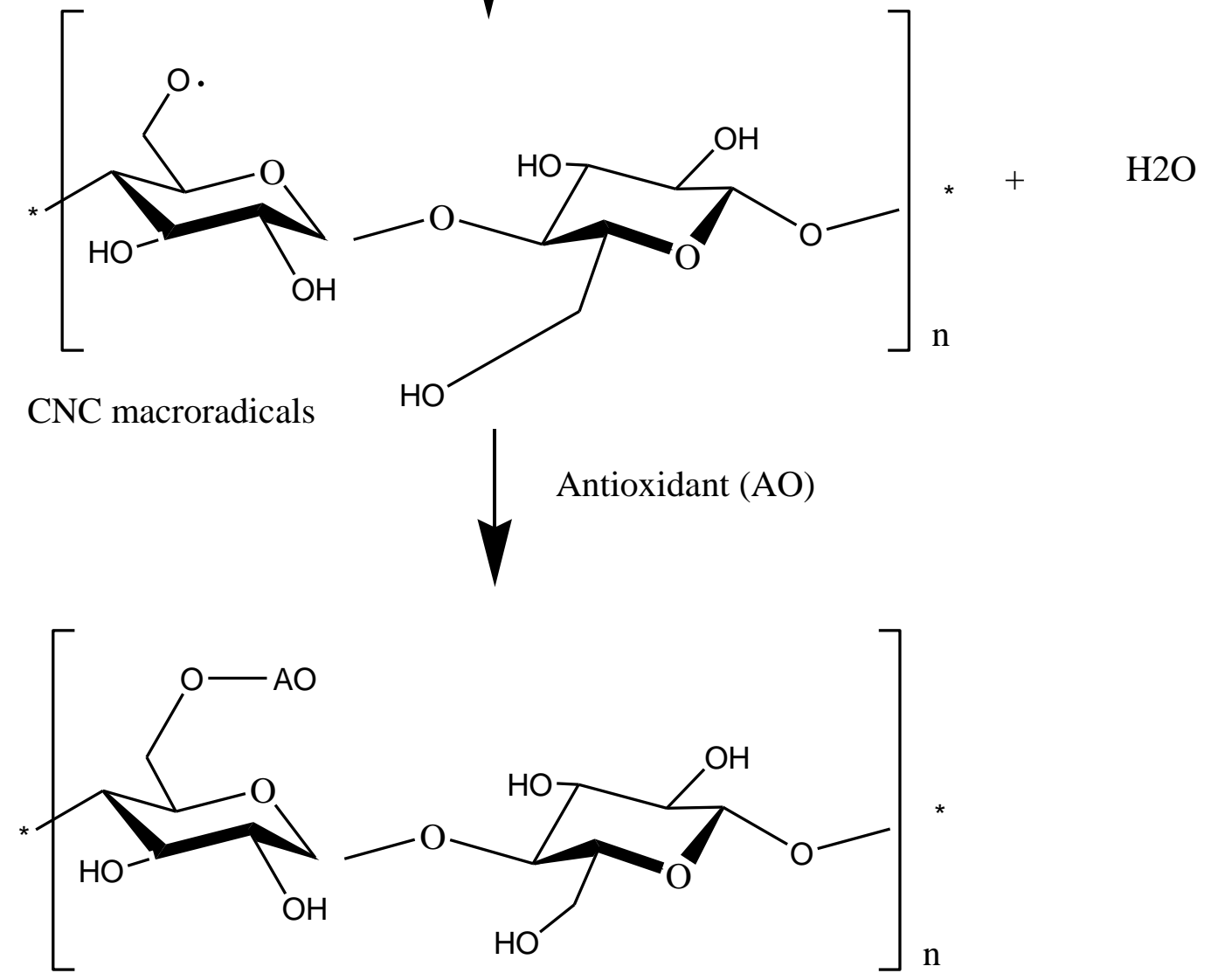

Fig. 2. The proposed mechanism of grafting procedure of antioxidant molecule on cellulose nanocrystals by action of redox pair. 


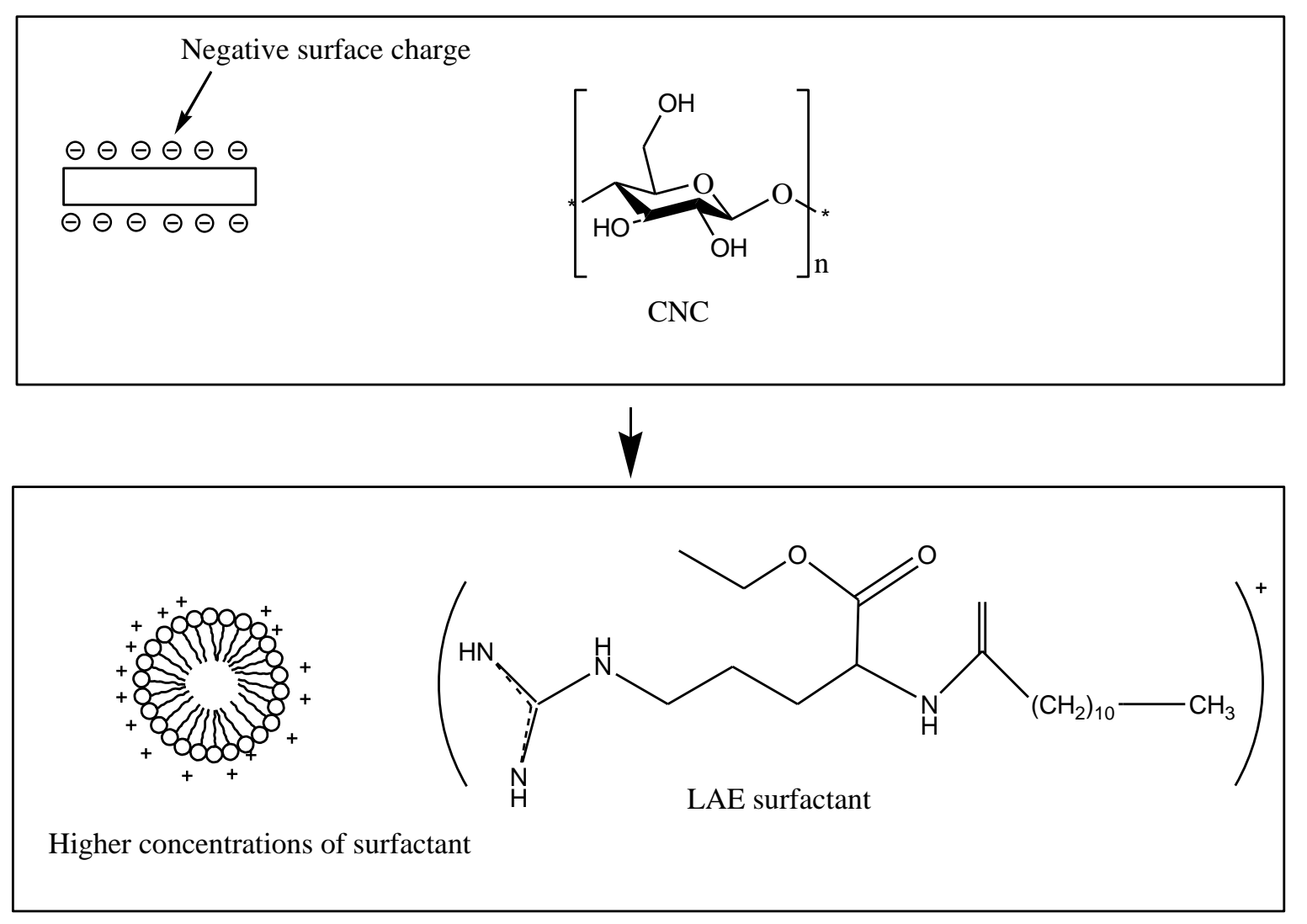

906

907

908

909

910

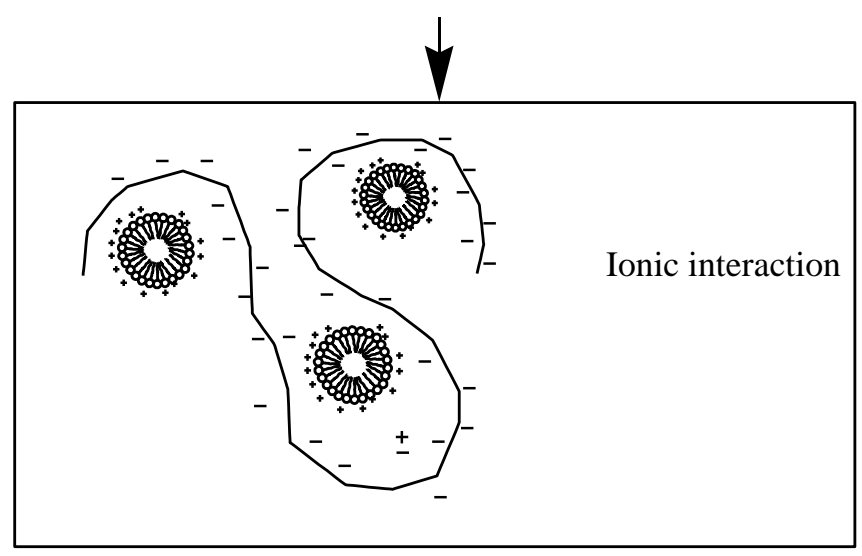

Fig. 3. Schematic representation of the ionic interaction of lauric arginate (LAE) surfactant micelles with CNC. 